\title{
EFFICACY OF TOPIRAMATE AS ADD-ON THERAPY IN TWO DIFFERENT TYPES OF PROGRESSIVE MYOCLONIC EPILEPSY
}

\author{
Caner Feyzi Demir', Hasan Hüseyin Özdemir ${ }^{2}$, Bülent Müngen ${ }^{1}$ \\ Department of Neurology, Firat (Euphrates) University, Elazıg, Turkey¹, Bismil State Hospital, Neurology, Diyarbakır, \\ Turkey ${ }^{2}$
}

Summary: The present article describes two unrelated cases of progressive myoclonic epilepsy (PME) of the Lafora's disease and Unverricht-Lundborg types who were treated with topiramate (TPM) as add-on therapy for their myoclonus. After the initiation of topiramate therapy both cases responded with marked decrease in myoclonic seizure frequency and improvement of quality of life. Topiramate appears to be a useful alternative agent in cases of PME and could be consider for adjunctive therapy.

Key words: Progressive myoclonic epilepsy; Topiramate

\section{Introduction}

Progressive myoclonus epilepsies are neurodegenerative disorders in which intractable myoclonic seizures, ataxia, cognitive impairment, and other neurologic deficits are prominent. More common entities or disease groups, Unverricht-Lundborg disease, Lafora's disease, neuronal ceroid lipofuscinoses, mitochondrial disorders, dentatorubral-pallidoluysian atrophy and sialidoses, account for the majority of PME cases in the world. Furthermore, a number of quite rare disorders can cause the PME phenotype (1).

Therapy is mainly symptomatic. Valproic acid, benzodiazepines, levetiracetam, and phenobarbital may be useful for seizures and myoclonus. Phenytoin, carbamazepine, oxcarbazepine, gabapentin, tiagabine, vigabatrin, and lamotrigine may aggravate myoclonus (2). There are some case reports, that was noted the effectiveness of topiramate in patients with PME $(3,4)$. Here, we present two cases of PME that successfully reduced the number and severity of seizures with TPM as add-on therapy.

\section{Case 1}

A 22-year-old woman was referred to our clinic because of pharmaco-resistant seizures. On presentation, the patient was observed to have nearly continuous myoclonic jerks. She had been diagnosed with Lafora disease in another medical center. The diagnosis was confirmed by the presence of multiple PAS-positive Lafora bodies in the ductal epithelial cytoplasms of the sweat glands with light microscopic examination of the axillary skin biopsy. At age 12 years learning difficulties was noted. She had a positive family history; her sister had the same symptoms and she died after 5 years of the onset of the disease. Medical history revealed that she had slight myoclonic jerks in her hands and face at first. Generalized tonic clonic seizures were added to the clinical picture 2 years after the myoclonic jerks which occurred at the age of 12 for the first time. Generalized tonic clonic seizures repeated one year later and later seizures occurred 3-4 times a year. The frequency of myoclonic jerks increased gradually and she began to suffer eight to ten drop attacks per day, causing difficulty in making her daily activities. The jerks progressively became more frequent and severe, so she appeared confused and unable to concentrate. She was still taking Na-valproate $(1500 \mathrm{mg} / \mathrm{d})$, lamotrigin $(100 \mathrm{mg} / \mathrm{d})$, clonazepam $(2 \mathrm{mg} / \mathrm{d})$ and levetiracetam $(1000 \mathrm{mg} / \mathrm{d})$ but seizures were not completely under the control. The seizures became more frequent and more severe, although she had been continued the antiepileptic medication regularly. During the next months, atypical absence seizures, myoclonic status and generalized convulsive status epilepticus occurred, and psychomotor slowing worsened. General analyses, routine laboratory analysis, serological tests, were normal. Levetiracetam was tapered off and discontinued. Topiramate was added to other medications and titrated to a mean dose of $125 \mathrm{mg} / \mathrm{d}$ in divided doses. Further adjustments of anticonvulsive treatment including topiramate led to markedly beneficial effects on seizures. Two weeks after the initiation of TPM, the frequency of myoclonic seizures decreased to 3-5 episodes per day. She has experienced no recurrence of generalized tonic-clonic seizure over more than six months.

\section{Case 2}

A 23-year-old woman with a long history of genetically confirmed Unverricht-Lundborg disease (EPM1) was referred to our clinic. Development was normal until 12 
years of age. Involuntary finger jerks, myoclonus were noted by age twelve. Her parents have noticed that her cognitive abilities have declined by age 13. Generalized tonic-clonic seizures (GTC) occurred upon awakening 1-2 times per month. Generalized myoclonus progressed with consequent stumbling or falling. Initially, stumbling while running, but by age 13 , even while walking. She was unable to walk without help (assistance) by age 18 . At the time of presentation, she was awkward in writing and had difficulty in eating without assistance. Mental deterioration was mild. Dysarthria was severe. Myoclonus appeared in all muscles, usually asynchronously but synchronously when exaggerated, and required her to grip the arms of her wheelchair to avoid flinging extremities or jumping up from the chair. Eye-opening, mental stress, photic or tactile stimuli, and intentional movements all activated myoclonus. Sleep suppressed myoclonus, but generalized myoclonus was marked upon awakening, and sometimes evolved to GTC. EEG evaluation showed widespread background anterior predominant slow activity with generalized spikewave or polyspike-wave discharges and photosensitivity in some records. She was treated with a combination of VPA (1500 mg/d), clonazepam (2 mg/d). Because electroencephalogram findings and cognitive performance deteriorated, and the frequency of seizures increased, topiramate was added and titrated to $100 \mathrm{mg}$ bid. Her epileptic symptoms improved, especially myoclonus decreased significantly. Her cognitive status and her dysarthria remained unchanged. Thirteen months following, she continued to have seizures, but she did not show worsening in seizures.

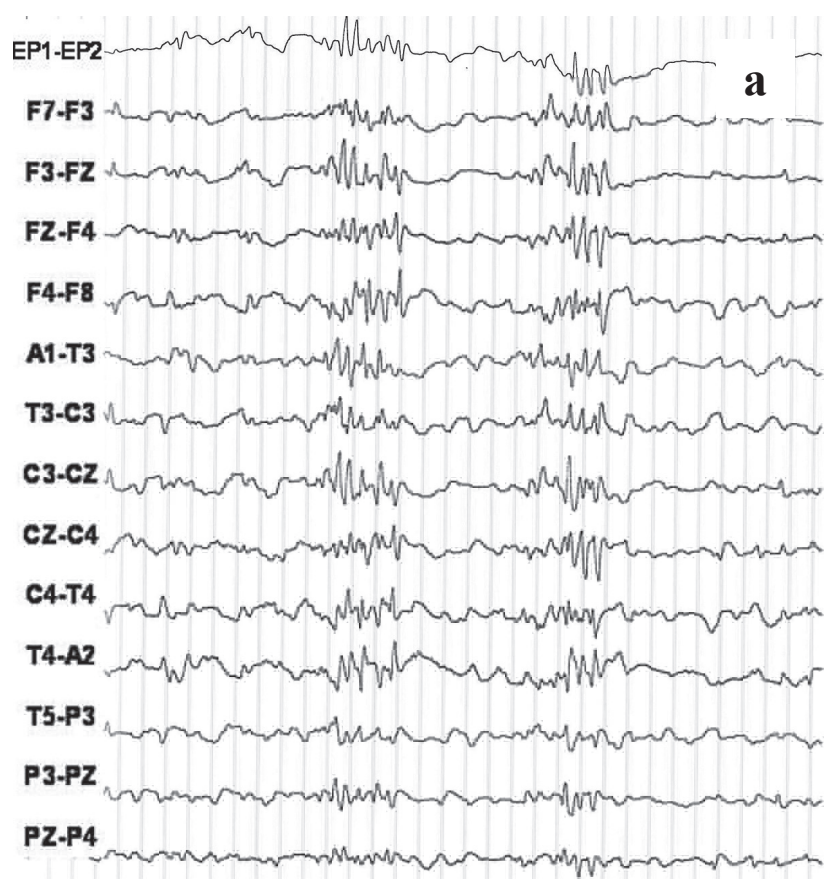

\section{Discussion}

We presented two genetically or pathologically confirmed different cases of PME in whom TPM had significant antiepileptic effect. Topiramate was originally used as an anticonvulsant. It is also indicated for the treatment of Lennox-Gastaut syndrome, prevention of migraines, bipolar disorder, borderline personality disorder, alcoholism, methamphetamine addiction, and obesity, post, infantile spasms. Several pharmacologic mechanisms of action of TPM have been identified and are thought to contribute to its anticonvulsant action $(5,6)$. These include; activation-dependent sodium channel-blocking effect, which reduces the duration and frequency of action, an augmentation of gamma-aminobutyrate acid activity at some subtypes of the GABA-A receptors, antagonism of AMPA/ kainate subtype of the glutamate receptor, and inhibition of the carbonic anhydrase enzyme, particularly isozymes II and IV (6-8). It has efficacy as add-on therapy in treatment-resistant patients with partial-onset and generalized seizures, with a good tolerability profile and minimal potential for interactions with other drugs.

A newer antiepileptic drug levetiracetam has also been reported to be effective in progressive myoclonic epilepsies (9). Beneficial effect of levetiracetam on seizures was not seen in our first case (with a dose of $1000 \mathrm{mg}$ twice daily). After discontinuation of levetiracetam with initiating topiramate, either myoclonic or generalized tonic clonic seizures were reduced. We observed only in one case of PME that TPM is more effective than LEV on seizures. It was demonstrated that topiramate has a negative impact

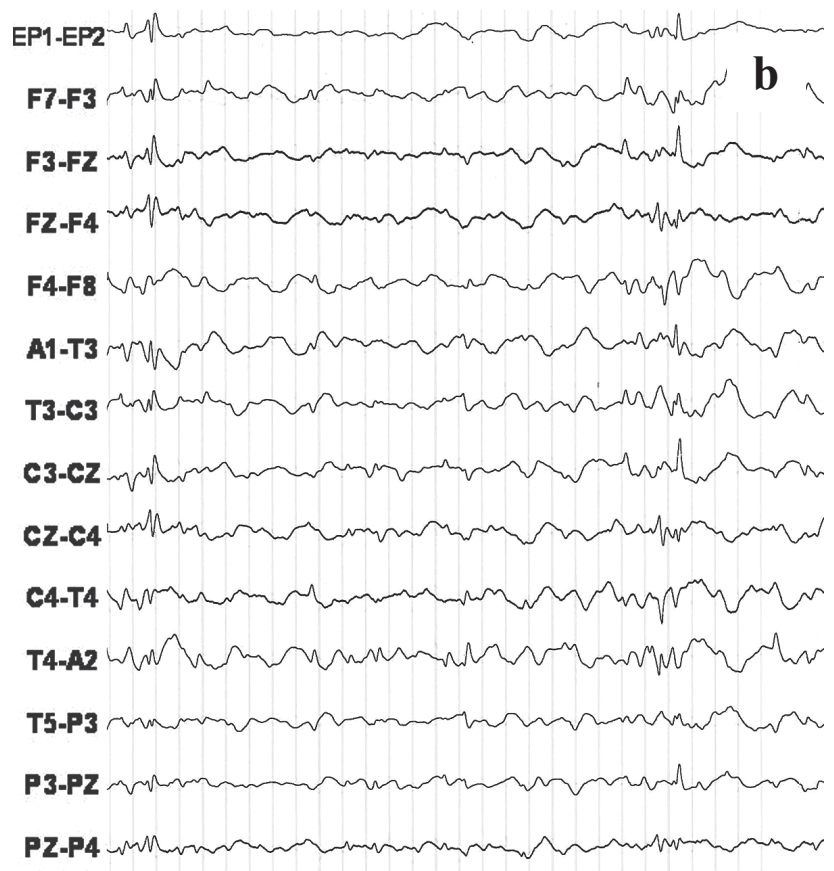

Figure: Interictal EEG samples of Case1: (a) before topiramate treatment, (b) after topiramate treatment 
in the patient's cognitive functions (10). Contrary to our expectations, we did not observe any significant cognitive deterioration in our first case; however, we observed partially cognitive improvement. We thought that, reduction in seizure frequency and severity may also improve patients' cognitive functions partially. It is thought that, cognitive improvement might be associated with reduction in seizure frequency and severity. Cognitive impairment was assessed as mild, no effects on daily routine activity; moderate, with need for some assistance from a caregiver; and severe; with total dependence in daily activity. Additionally in first case, partially suppression of paroxysmal epileptiform discharges (duration of discharges was shortened, amplitude of discharges was decreased, interval of discharges was delayed) was observed after TPM administration (Figure a, b). However, there was no change in EEG rhythm or amplitude during TPM administration in the second case.

In conclusion, in an open naturalistic setting topiramate used in low dose and individualized titration was effective and well tolerated in add-on therapy $(5,11)$. There are anecdotal and individual case reports that TPM may have utility in treating seizures of PME (3). Our experience is consistent with previous reports that TPM is useful in different types of PME. TPM could be considered as an effective treatment option against different types of seizures in cases of progressive myoclonic epilepsy. However, even in the short term it helped improve the patient's symptoms and quality of life. The beneficial effect of reducing seizures may offset the adverse cognitive effects. Prospective controlled studies are needed to assess its long-term usefulness in patients with PME.

\section{References}

1. Lehesjoki AE. Molecular background of progressive myoclonus epilepsy. EMBO J. $2003 ; 15 ; 22(14): 3473-3478$.

2. Neubauer BA. Progressive and Infantile Myoclonic Epilepsies. In: Wyllie E, ed. The Treatment of Epilepsy: Principles and Practice. (5th ed.) Philadelphia: Wolters Kluwer/Lippincott Williams \& Wilkins. 2011; 269-279.

3. Aykutlu E, Baykan B, Gürses C, et al. Add-on therapy with topiramate in progressive myoclonic epilepsy. Epilepsy Behav. 2005; 6(2): 260-263.

4. Fallah R. Topiramate as a new antiepileptic drug in epileptic children in Iran. Indian J Pediatr. 2006; 73(12): 1073-1075.

5. Wauquier A, Zhou S. Topiramate: a potent anticonvulsant in the amygdala-kindled rat. Epilepsy Res 1996; 24: 73-77.

6. White HS. Clinical significance of animal seizure models and mechanism of action studies of potential antiepileptic drugs. Epilepsia 1997; 38(suppl 1): 9-17.

7. Biton V, Montouris GD, Ritter F, et al. A randomized, placebo controlled study of topiramate in primary generalized tonic-clonic seizures: Topiramate YTC Study Group. Neurology. 1999; 52: 1330-1337.

8. Stephen LJ, Sills GJ, Brodie MJ. Topiramate in refractory epilepsy: a prospective observational study. Epilepsia. 2000; 4: 977-980.

9. Crest C, Dupont S, Leguern E, Adam C, Baulac M. Levetiracetam in progressive myoclonic epilepsy: an exploratory study in 9 patients. Neurology. 2004; 24; 62(4): 640-643.

10. Thompson PJ, Baxendale SA, Duncan JS, Sander JW. Effects of topiramate on cognitive function. J Neurol Neurosurg Psychiatry 2000; 69: 636-641.

11. Shank RP, Gardocki JF, Streeter AJ, et al. An overview of the preclinical aspects of topiramate: pharmacology, pharmacokinetics, and mechanism of action. Epilepsia 2000; 41(suppl. 1): 3-9.

Received: $10 / 11 / 2012$

Accepted in revised form: 17/01/2013

\section{Corresponding author:}

Hasan Hüseyin Özdemir, M.D., Bismil State Hospital, Neurology, Diyarbakır, Turkey; e-mail: drhasanh@gmail.com 